\title{
Effect of ambient air quality on exacerbation of COPD in patients and its potential mechanism
}

This article was published in the following Dove Press journal:

International Journal of Chronic Obstructive Pulmonary Disease

Peng Yan'
Pengfei Liu'
Rong Lin ${ }^{2}$
Kun Xiao
Sheling Xie
Kaifei Wang
Yuhan Zhang
Xanxue He ${ }^{2}$
Shifeng Zhao'
Xingang Zhang'
Martin Liu ${ }^{4}$
Lixin Xie'
'Pulmonary and Critical Care Medicine,
The General Hospital of PLA, Beijing
I00853, People's Republic of China;
${ }^{2}$ Medical School of Chinese PLA, Beijing
I00853, People's Republic of China;
${ }^{3}$ Department of Respiratory Disease,
The Sanya People's Hospital, Sanya
572000, People's Republic of China;
${ }^{4}$ Pulmonary, Critical Care, Sleep and
Allergy, Department of Internal Medicine,
University of Nebraska Medical Center,
Omaha, NE 68I98-59I0, USA

Omaha, NE 68198-5910, USA
Correspondence: Lixin Xie

Department of Respiratory Diseases, The General Hospital of PLA, Beijing 100853,

People's Republic of China

Tel +86I 39| I25 2807

Email xielx30I@126.com
Background: Chronic obstructive pulmonary disease (COPD) is a disease of continuous progress and environmental factors may affect the progress. COPD patients' activity tolerance and quality of life are associated with air quality. COPD exacerbation from the perspective of geographical air quality has not been reported.

Objectives: To explore environmental effect of two different geographical places on COPD exacerbation and the effect of cigarette smoke extract and carbon particles on bronchial epithelial cell viability.

Methods: Total 139 COPD patients, who lived in Beijing during summer and temporarily migrated to Sanya city in winter, have been enrolled. Respiratory symptoms and lung function data were collected when they were living in Beijing or Sanya, respectively. Effect of cigarette smoke extract plus ultrafine carbon particles on airway epithelial cells were studied.

Measurements and main results: Air pollution as measured by air quality index (AQI) in Beijing summer $(113.1 \pm 14.2)$ was significantly worse than that in Sanya winter (49.4 $\pm 8.9, p<0.001$ ). The COPD Assessment Test (CAT) score was significantly higher in Beijing (26.4 \pm 7.1$)$ than that in Sanya $(20.0 \pm 8.0, p=0.019)$. Modified Medical Research Council dyspnea scale was also significantly higher in Beijing (2.9 \pm 0.9$)$ than that in Sanya $(1.9 \pm 0.8, p<0.001) . \mathrm{FEV}_{1}$ was significantly improved when the patients were in Sanya $(48.88 \pm 24.78 \%)$ compared to that in Beijing $(41.79 \pm 20.06 \%, p<0.01)$. Compared with Beijing and Sanya, the relative risk (RR) of hospitalization and acute exacerbation were 1.64 and 3.36, respectively. In vitro study demonstrated that apoptosis of BEAS2B cells in response to cigarette smoke extract plus ultrafine carbon particles $(25.50 \pm 2.10 \%)$ was significantly higher than that of control culture $(2.30 \pm 1.05 \%, p<0.01)$.

Conclusion: These findings suggested that ambient air pollution cause COPD exacerbation, and that air pollutants particle matters induce apoptosis of airway epithelial cells.

Keywords: chronic obstructive pulmonary disease, temporary migration, air pollution, lung function, apoptosis of airway epithelial cells

\section{Introduction}

With rapid industrialization and economic growth over the last decade, air pollution has become a serious issue in China. Especially, Beijing has been one of the several cities with deteriorating air quality. It this regard, in the last decade, Beijing experienced multiple prolonged periods of severe smog with extremely high ambient $\mathrm{PM}_{2.5}$ (Particles having a diameter of less than or equal to $2.5 \mu \mathrm{m}$ ) concentration. ${ }^{1}$ In contrast, Sanya city, a city in Hainan Island, China, has been a city with top quality air in China. Therefore, Sanya city becomes a preferred place to live for senior Chinese citizens and patients with respiratory diseases during the winter season. 
The adverse health effects of air pollution have been reported in many epidemiological studies. ${ }^{2-4}$ Approximately, 3.1 million deaths were associated with ambient particulate matter pollution worldwide in 2010. ${ }^{5}$ Toxicological studies indicated that air pollution, especially, the exposure of particle matters with diameter $2.5 \mu \mathrm{m}$ or less $\left(\mathrm{PM}_{2.5}\right)$ can cause lung inflammation and suppression of pulmonary immune function. ${ }^{6}$ Studies have also demonstrated the association of air pollution and respiratory disease ${ }^{1,7}$ or exacerbation of COPD and asthma. ${ }^{8,9}$ However, to our knowledge, no study has reported on the association of ambient air pollution and COPD exacerbation in the patients who lived in a city with heavy air pollution and temporarily migrated to another city with top quality air in China. The current study was, therefore, designed to explore the association of ambient air quality and COPD exacerbation by investigating respiratory symptoms and lung functions in COPD patients who lived in Beijing during summer and temporarily migrated to Sanya during winter season, a subtropical city, in Hainan Island, China. Furthermore, potential mechanism of air pollutants effect on COPD exacerbation was investigated by exposing an airway epithelial cell line BEAS2B cells to cigarette smoke and ultrafine carbon particles.

\section{Materials and methods}

\section{Environmental data collection from Sanya and Beijing}

Daily data of ambient air quality of Beijing and Sanya were obtained from the following website: http://www. pm25.in/beijing and http://www.pm25.in/sanya. Daily temperature and humidity in Beijing and Sanya were obtained from the following website: http://www.cma. gov.cn.

\section{Patient enrollment}

Patients who were at the GOLD (2014) II IV of chronic obstructive pulmonary disease (COPD) were selected to be potential subjects for the current study. After obtaining consent forms, a total of 139 COPD patients, who lived in Beijing from April through September (late spring to early fall) and temporary lived in Sanya from October through March (late fall to early spring) every year for the last 3 years or longer, were finally enrolled into the current study. Among the 139 patients with COPD, the proportion of moderate, severe and extremely severe COPD patients was $21 \%$ (29), 39\% (54) and 40\% (56), respectively. The patients' treatment used was constantly same in both places. The study protocol was approved by the Ethic Committee of The General Hospital of People's Liberation Army, China (\#S2014-099).

1. Inclusion criteria: patients with $<80 \%$ predicted $\mathrm{FEV}_{1}$, and $<70 \%$ of $\mathrm{FEV}_{1} / \mathrm{FVC}$, at stable phase of COPD, and willing to sign the consent form.

2. Exclusion criteria: patients at exacerbation of COPD, or with comorbidity of asthma, tuberculosis, diffuse pan-bronchiolitis; patients who could not perform 6 mins walking test; patients with multi-organ dysfunction.

Patients who were enrolled into this study regularly visited The Hainan Branch of The PLA General Hospital or Sanya People's Hospital for free lung function examination.

This study was conducted in accordance with the Declaration of Helsinki. We have no deidentified participant data to share.

\section{Clinical evaluation and pulmonary function test}

Patients' symptoms and breathless severity were acquired by filling the publically available "COPD Assessment Test (CAT)" scorecard and "Modified Medical Research Council (MMRC)" dyspnea scale card. An exacerbation of COPD was defined as an acute event characterized by a worsening of the patient's respiratory symptoms (baseline dyspnea, cough and/or sputum production) that was beyond normal day-to-day variations and leads to a change in medication.

Pulmonary function test (PFT) was performed by certified PFT technician using Master Screen PFT (Jaeger, Germany).

\section{In vitro effect of cigarette smoke extract and carbon particles on bronchial epithelial cells}

1. Cell culture: BEAS2B cells were purchased from ATCC, https://www.atcc.org/search\#q=beas$2 \mathrm{~b} \&$ sort $=$ relevancy. Cells were cultured with LHC9 medium supplemented with $1 \%$ fetal calf serum (FCS), penicillin/streptomycin and fungizon (Life Technology, Shanghai, China).

2. Cigarette smoke extract (CSE) preparation: CSE was prepared with a modification of the method of Carp and Janoff. ${ }^{10}$ Briefly, one 100-mm research grade cigarette without filter (University of Kentucky, 
USA) was combusted through $15 \mathrm{~mL}$ distilled water a Variable Speed Pump (Fisher Scientific, Pittsburgh, USA). The resulting suspension was filtered through a $0.22-\mu \mathrm{m}$ pore filter (Lida Manufacturing Corp., Kenosha, WI, USA). This solution was considered to be $100 \%$ CSE and diluted with LHC-9 medium as desired.

3. Cell treatment: Cells were treated with either $10 \%$ CSE or $5 \mu \mathrm{g} / \mathrm{mL}$ ultra fine carbon particles (UFCP), or both in the LHC-9 medium. Cells cultured in LHC-9-medium-only were served as control.

4. TUNEL assay: TUNEL assay was performed using the fluorescence-labeled TUNEL assay kit (Roche Molecular Biochemicals, USA). Briefly, BEAS2B cells were cultured in collagen-coated 8-chamber slides till confluence. Cells were fixed with formalin and permeabilized with $0.1 \%$ Tritone $\mathrm{X}-100$ (in $0.1 \%$ sodium citrate) for 2 mins on ice and rinsed with PBS. The cells were then reacted with the TUNEL mixture at $37^{\circ} \mathrm{C}$ for 60 mins. After washing, cells were counter stained with $1 \mu \mathrm{g} / \mathrm{mL}$ DAPI for 5 mins. The fluorescence incorporation into nucleotide was detected and photographed under fluorescence microscopy. TUNEL positive cells vs total cell number was counted.

5. Immunoblotting: After exposure to desired reagents, BEAS2B cells were lysed with lysing buffer (Thermo Fisher China, Shanghai, China). After brief sonication, the cell lysate was centrifuged at $12,000 \mathrm{~g}$ for $10 \mathrm{mins}$ at $4^{\circ} \mathrm{C}$. Protein concentrations in the supernatant were determined by a protein-binding assay (Thermo Fisher China, Shanghai, China). After heating at $95^{\circ} \mathrm{C}$ for 5 mins and rapid cooling on ice, $10 \mu \mathrm{g}$ of total protein was mixed with $2 \times$ sample loading buffer and loaded into each well of Mini-protein 3 Cell System (Bio-Rad, Life Science, Beijing, China). After electrophoresis, the proteins were transferred to PVDF membrane with the semi-dry electrophoretic transfer system (Bio-Rad, Life Science, Beijing, China). The membrane was blocked with $5 \%$ nonfat milk in PBS-tween at room temperature for $1 \mathrm{hr}$ and then exposed to primary antibodies at $4^{\circ} \mathrm{C}$ overnight. Target proteins were subsequently detected using horseradish peroxidase-conjugated 2nd antibody and with an enhanced chemiluminescence detection system.
6. ELISA: Quantification of the cytokines (IL-1ß, IL-6, IL-8, TNF- $\alpha$ and TGF- $\beta 1$ ) and matrix metalloproteinases (MMP-1 and MMP-9) were performed using ELISA Duoset and following the manufacturer's instruction (R\&D Systems China, Shanghai, China).

\section{Statistical analysis}

Each experiment was repeated at least three times. All quantitative data were expressed as mean \pm SD. Comparison of paired data was performed using the Student $t$-test, whereas multi-group data were analyzed by ANOVA followed by Tukey correction using PRISM 6 software. $P<0.05$ was considered significant.

\section{Results}

Total of 139 COPD patients were enrolled into the current study. Of them, 48 were male and 91 were female, with average age of $71.39 \pm 10.07$ years old. These patients lived in Beijing for most of their life and temporarily migrated to Sanya City, Hainan Island, during the winter season at least for 3 consecutive years. As shown in Figure 1, during winter season in Sanya city, temperature was above $20^{\circ} \mathrm{C}$ with average of $23.1 \pm 4.2^{\circ} \mathrm{C}$, which was similar to that of summer weather in Beijing (24.3 $\pm 6.7, p=0.674$, Figure 1A). Humidity, however, was higher in Sanya winter (90.6 $\pm 4.9 \%)$ than that in Beijing summer $(58.0 \pm 10.0 \%, p=0.001$, Figure 1B). In contrast, air pollution as measured by air quality index (AQI) in Beijing summer $(113.1 \pm 14.2)$ was significantly worse than that in Sanya winter (49.4 \pm 8.9 , $p<0.001$, Figure 1C).

Patients visited Sanya Hospital, Affiliated to The General Hospital of PLA when they were living in Sanya during the winter season, and visited The General Hospital of PLA in Beijing when they returned to Beijing during the summer season. As shown in Figure 2, the comparison of COPD Assessment Test (CAT) score for the same patients in two different cities indicated that CAT score was significantly higher in Beijing (26.4 \pm 7.1$)$ than that in Sanya (20.0 $\pm 8.0, p=0.019$, Figure 2B). Similarly, Modified Medical Research Council (MMRC) dyspnea scale was also significantly higher in Beijing (2.9 \pm 0.9$)$ compared to that in Sanya $(1.9 \pm 0.8, p<0.001$, Figure 3$)$.

Of the 139 COPD patients, 89 patients completed pulmonary function test (PFT) in both places. It was found that forced expiratory volume in 1 second $\left(\mathrm{FEV}_{1}\right)$ for same patients was significantly improved when the patients were in Sanya $(48.88 \pm 24.78 \%)$ compared to that 


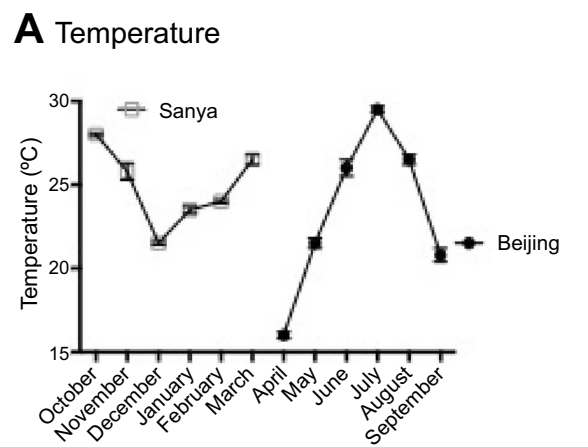

B Humidity

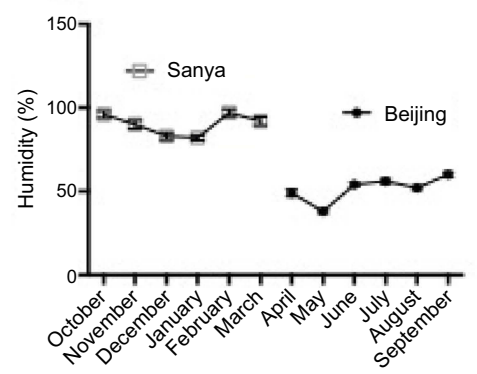

C Air quality index

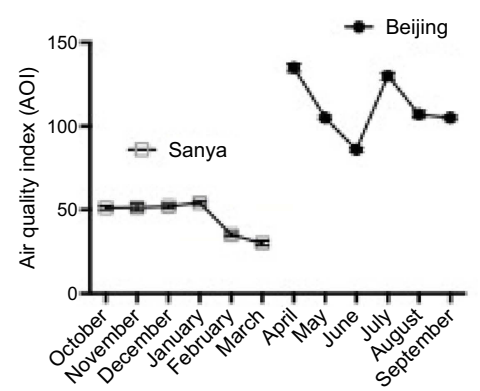

Figure I Comparison of temperature, humidity and ambient air quality between Beijing and Sanya. Monthly average temperature, humidity and air quality data were obtained from the Meteorological Department of Beijing City and Sanya City. (A) Monthly average of temperature. (B) Monthly average of humidity. (C) Monthly average of air quality in Beijing and Sanya.

A

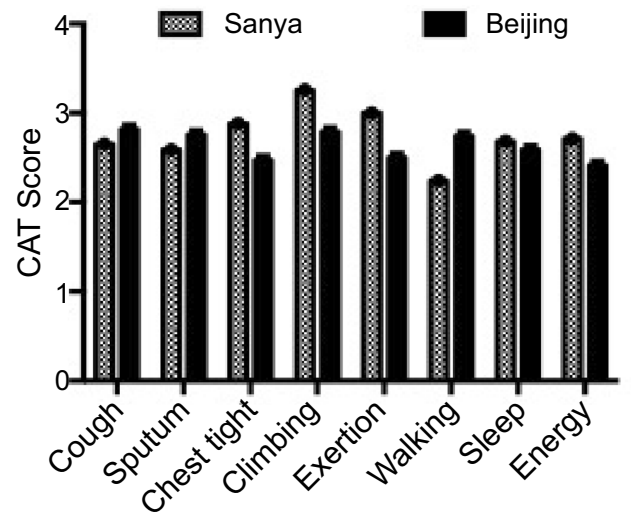

B

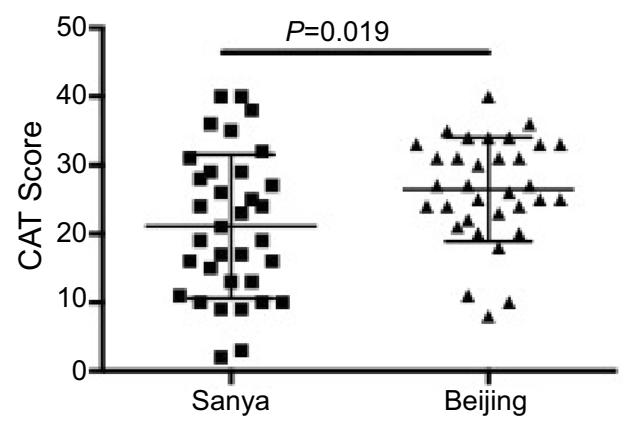

Figure 2 Comparison of COPD Assessment Test (CAT) score in patients when they were in Beijing or Sanya. (A) CAT score of common symptoms. (B) Average CAT score of the patients in Beijing or Sanya.

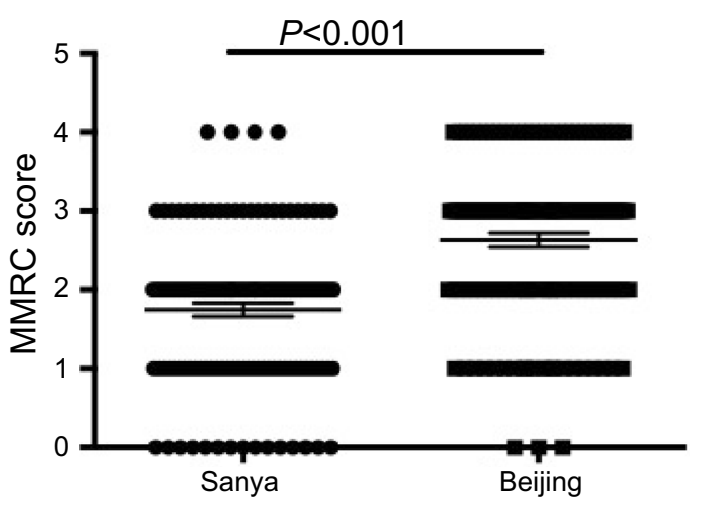

Figure 3 Comparison of Modified Medical Research Council (MMRC) dyspnea scale.

in Beijing (41.79 $\pm 20.06 \%, p<0.01$, Figure 4A). Similarly, other PFT parameters including forced vital capacity (FVC), maximum expiratory flow (MEF) and 50\% vital capacity maximal expiratory flow $\left(\mathrm{MEF}_{50}\right)$ or $25 \%$ vital capacity maximal expiratory flow $\left(\mathrm{MEF}_{25}\right)$ were also significantly improved when the patients were in Sanya in comparison to that in Beijing (Figure 4B-E). Consistent with the findings of lung function status, COPD acute exacerbation rate and hospitalization rate were significantly higher in the patients when they were living in Beijing (188.49\% acute exacerbation rate and $51.80 \%$ hospitalization rate) compared to that of living in Sanya (56.12\% and $31.65 \%$, respectively, Table 1$)$. When the patients returned to Beijing from Sanya, the relative risk of the hospital admission was 1.64; and the relative risk of acute exacerbation was 3.36 (Table 1).

In order to explore potential mechanisms of air pollutants on COPD exacerbation, in vitro cell culture model was utilized to investigate the effect of particle matters and cigarette smoke on bronchial epithelial cells. As shown in Figure 5, either 10\% cigarette smoke extract (CSE) or $5 \mu \mathrm{g} / \mathrm{mL}$ UFCP induced apoptosis in BEASE2 cells as evidenced by positive TUNEL 


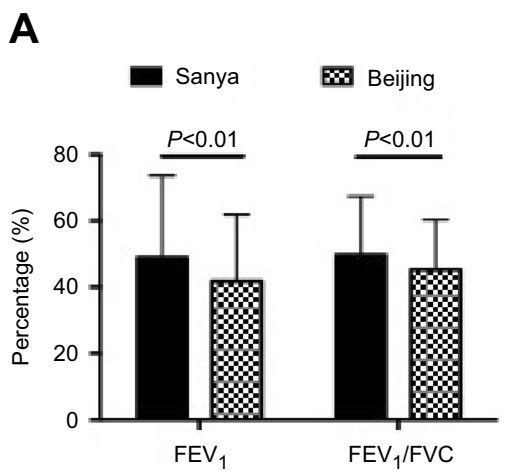

B

C
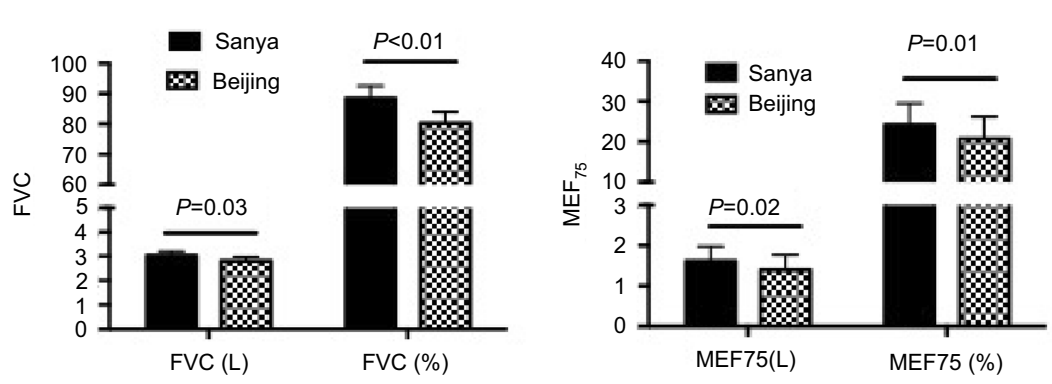

D

\section{E}
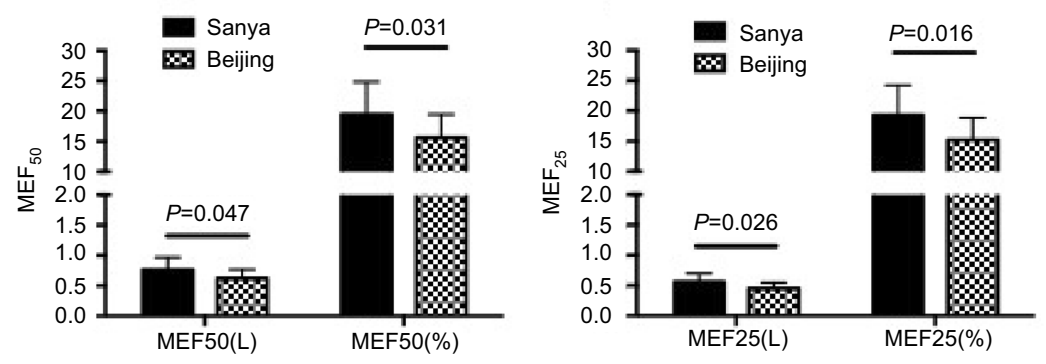

Figure 4 Comparison of pulmonary function test. Patients' pulmonary function was tested when they were in Beijing or Sanya. $(\mathbf{A}) \mathrm{FVE}_{1}$ or $\mathrm{FEV}_{\mathrm{I}} / \mathrm{FVC}$. (B) $\mathrm{FVC}$. $(\mathbf{C}) \mathrm{MEF}_{75}$. (D) $\mathrm{MEF}_{50}$. (E) $\mathrm{MEF}_{25}$.

Abbreviations: MEF, maximum expiratory flow; L, liter.

Table I Relative risk (RR) of hospitalization and acute exacerbation

\begin{tabular}{|l|l|l|}
\hline & $\begin{array}{l}\text { Beijing } \\
(\mathbf{N}=139)\end{array}$ & $\begin{array}{l}\text { Sanya } \\
(\mathbf{N}=139)\end{array}$ \\
\hline Hospitalization times (\%) & $72(51.80)$ & $44(31.65)$ \\
Acute exacerbation times (\%) & $262(188.49)$ & $78(56.12)$ \\
\hline
\end{tabular}

Notes: $R$ R of hospitalization $=51.80 / 31.65=1.64$. RR of acute exacerbation $=188.49 / 56.12=3.36$.

staining (Figure 5A), which was statistically significant (Figure 5B, 10.42 $\pm 1.42 \%$ of CSE alone, and $15.42 \pm 1.21 \%$ of UFCP alone versus $2.30 \pm 1.05 \%$ of control, $p<0.05)$. CSE plus UFCP further resulted in higher rate of positive TUNEL staining (Figure 5B, 25.50 $\pm 2.10 \%, p<0.01$ compared to control). Consistent with the findings of TUNEL staining, $10 \%$ CSE and $5 \mu \mathrm{g} / \mathrm{mL}$ UFCP significantly stimulated caspase-3 expression, but did not affect caspase-9 level (Figure 6A-C). Furthermore, CSE and UFCP significantly inhibited Bcl-2 protein expression (Figure 6A and D).

In addition to apoptosis induction, cigarette smoke and particle matters stimulated IL-1ß production by the BEAS2B cells $(34.1 \pm 2.5 \mathrm{pg} / \mathrm{mL}$ of $\mathrm{CSE}$ and $30.7 \pm 2.2$ $\mathrm{pg} / \mathrm{mL}$ of UFCP versus $16.6 \pm 3.0 \mathrm{pg} / \mathrm{mL}$ of control, $p<0.05$, Figure 7A). Furthermore, CSE plus ultrafine carbon black particle could further stimulate IL- $1 ß$ production (55.9 $\pm 4.0 \mathrm{pg} / \mathrm{mL}, p<0.01$ compared to CSE or UFCP alone, Figure 7A). While neither CSE nor UFCP alone had significant effect on IL-6 $(102.1 \pm 9.7 \mathrm{pg} / \mathrm{mL}$ of CSE and $120.3 \pm 13.0 \mathrm{pg} / \mathrm{mL}$ of UFCP versus $80.1 \pm 9.1 \mathrm{pg} / \mathrm{mL}$ of control, $p>0.05$, Figure $7 \mathrm{~B})$ or IL-8 $(220.4 \pm 78.3 \mathrm{pg} / \mathrm{mL}$ of $\mathrm{CSE}$ and $314.9 \pm 24.2 \mathrm{pg} / \mathrm{mL}$ of UFCP versus $152.7 \pm 13.3$ $\mathrm{pg} / \mathrm{mL}$ of control, $p>0.05$, Figure $7 \mathrm{C}$ ) production, CSE plus UFCP significantly stimulated IL-6 (176.4 $\pm 7.2 \mathrm{pg} /$ $\mathrm{mL}, p<0.05$, Figure $7 \mathrm{~B})$ or IL-8 production $(363.3 \pm 66.9$ $\mathrm{pg} / \mathrm{mL}$, Figure 7C). In contrast, total TGF- 31 production was significantly inhibited in the presence of CSE $(902.0$ $\pm 68.0 \mathrm{pg} / \mathrm{mL})$ or UFCP $(934.4 \pm 3.9 \mathrm{pg} / \mathrm{mL}$ versus 1296.0 $\pm 61.0 \mathrm{pg} / \mathrm{mL}$ of control, $p<0.05$, Figure 7D), and the suppression was further augmented in the presence of CSE plus UFCP $(436.7 \pm 13.9 \mathrm{pg} / \mathrm{mL}, p<0.01$, Figure 7D). In addition, neither CSE nor UFCP had significant effect on TNF- $\alpha$, MMP-1 or MMP-9 production by the cells (data not shown). 
A: TUNEL assay

Control
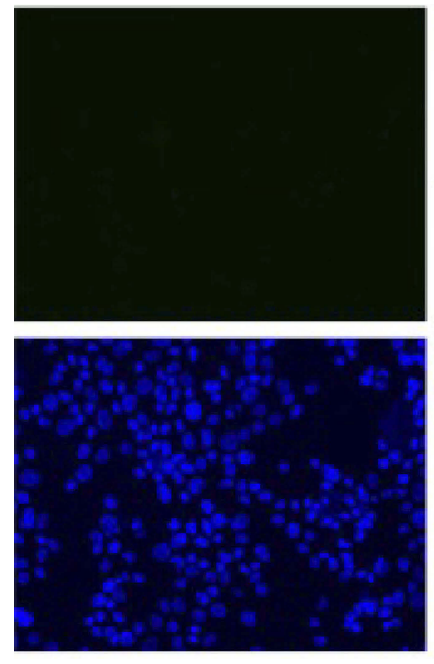

$10 \% \mathrm{CSE}$
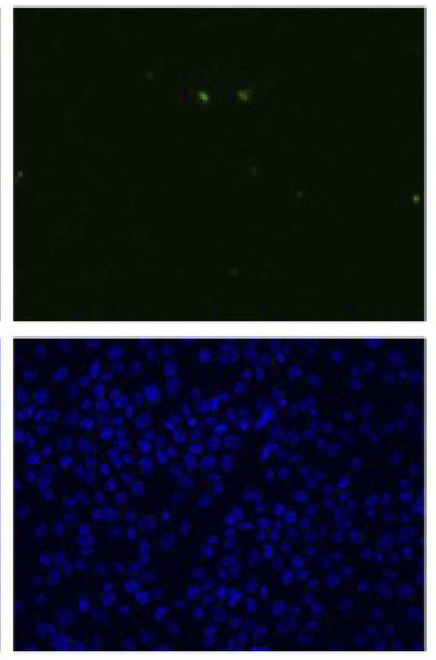

$5 \mu \mathrm{g} / \mathrm{mL}$ UFCP
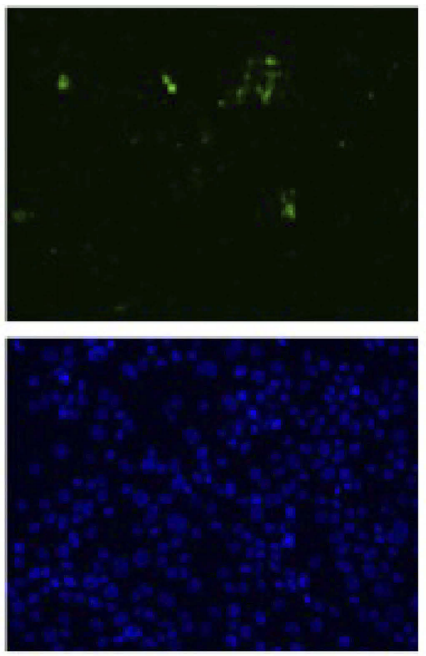

$10 \%$ CSE + UFCP
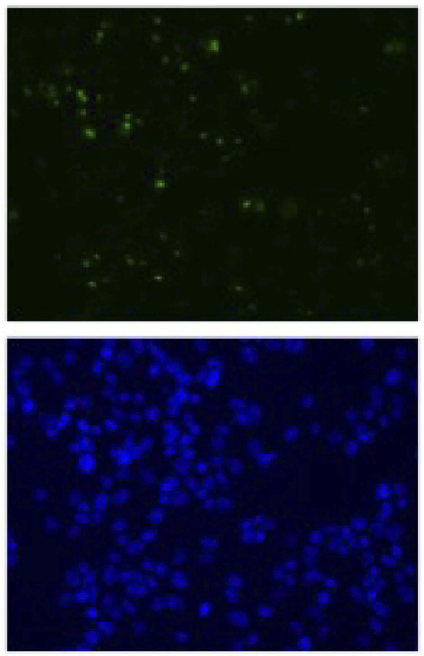

B

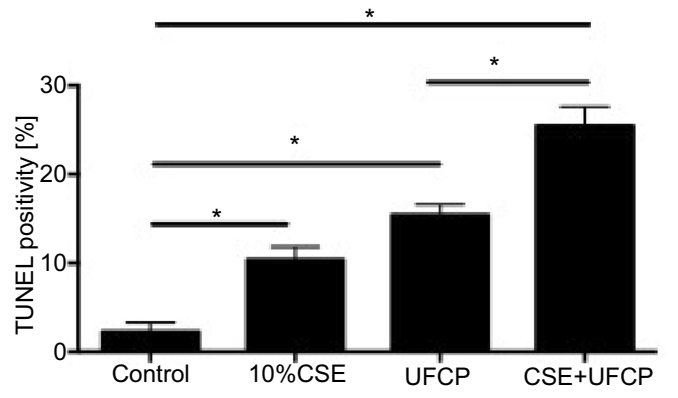

Figure 5 Effect of CSE and UFCP on BEAS2B cell viability. BEAS2B cells were exposed to 10\% cigarette smoke extract (CSE) and/or ultrafine carbon particles (UFCP) for 24 hrs. TUNEL assay was performed as described in the methods. (A) Representative image of TUNEL staining. Green: TUNEL positive staining; Blue: nuclei. (B) Quantitative graph of TUNEL positivity. Horizontal axis: treatment of the cells; vertical axis: TUNEL positivity in 5 high power field. $*_{p}<0.05$.

\section{Discussion}

The current study longitudinally compared COPD patients' symptoms and lung functions when they were living in two different cities, where ambient air quality and relative humidity were significantly different. We found that COPD patients' symptoms were reduced, lung function was improved and exacerbation rate and hospitalization rate were significantly decreased when the patients temporarily migrated to Sanya city, a city with best air quality and higher relative humidity in China, compared with that in Beijing, the Capital city with heavy air pollution and relatively dry city. We further demonstrated that BEAS2B cells underwent significant apoptosis in response to UFCP and CSE, and that $\mathrm{Bcl}-2$ was significantly decreased while caspase-3 was significantly increased in response to UFCP and CSE. CSE and/or UFCP also stimulated synthesis of inflammatory cytokines (IL-1ß, IL-6 and IL-8), but significantly inhibited TGF-ß1 synthesis by the BEAS2B cells. These findings suggested that ambient air quality be associated with COPD exacerbation, and that $\mathrm{PM}_{2.5}$ and cigarette smoke cause airway cellular injury through inducing airway epithelial cell apoptosis as well as stimulating airway inflammation.

During the last decade, Beijing has experienced heavy air pollution, especially in winter and early spring, because of the coal burning and wind-blown dust from the surrounding industrial cities as well as the vehicle exhaust from exponential growing number of automobiles. ${ }^{11}$ In this regard, it has been reported that the peak hourly concentration of ambient $\mathrm{PM}_{2.5}$ in Beijing was up to $800 \mu \mathrm{g} / \mathrm{m}^{3}$, and the average ambient $\mathrm{PM}_{2.5}$ concentration in 2013 reached $89.5 \mu \mathrm{g} / \mathrm{m}^{3}{ }^{1}$ The 
A
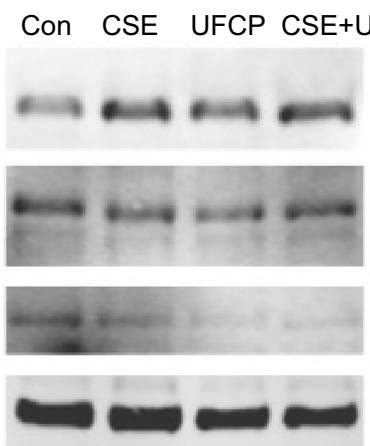

C Caspase-9

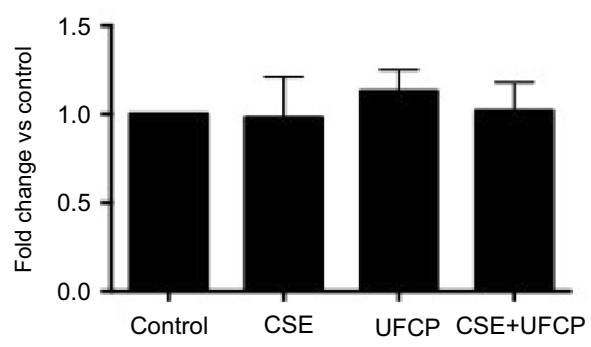

B Caspase-3

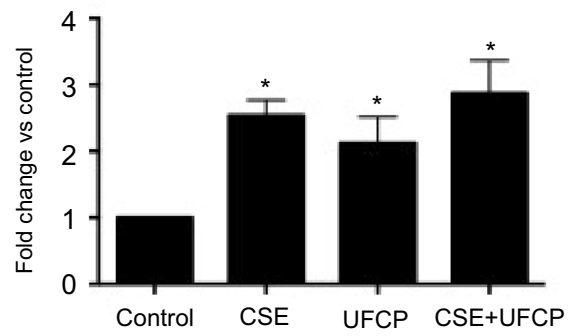

D $\mathrm{Bcl}-2$

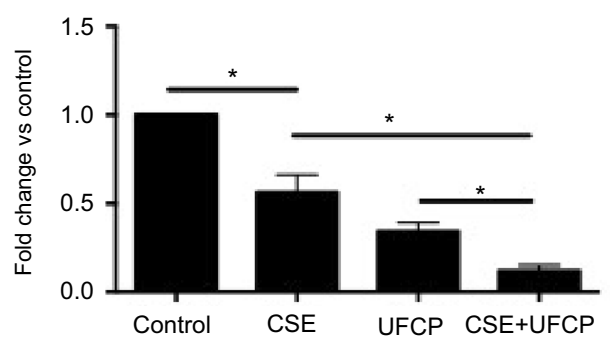

Figure 6 Immunoblotting of caspase-3, caspase-9 and Bcl-2. BEAS2B cells were exposed to 10\% cigarette smoke extract (CSE) and/or ultrafine carbon particle (UFCP) for 24 hrs. Cells were harvested with cell lysis buffer and subjected for immunoblotting as described in the methods. (A) Representative image of the immunoblotting. (B) Semiquantification of caspase-3. ${ }^{*} p<0.05$ compared to control. (C) Semi-quantification of caspase-9. (D) Semi-quantification of Bcl-2. $* p<0.05$.

A IL-1ß

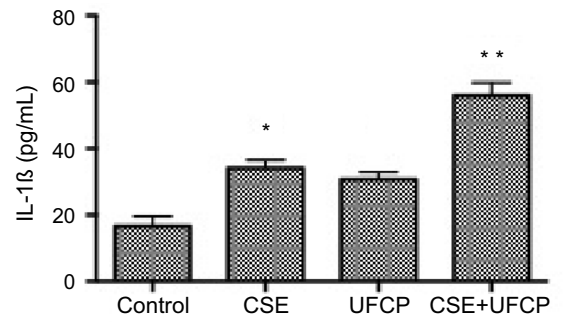

C IL-8

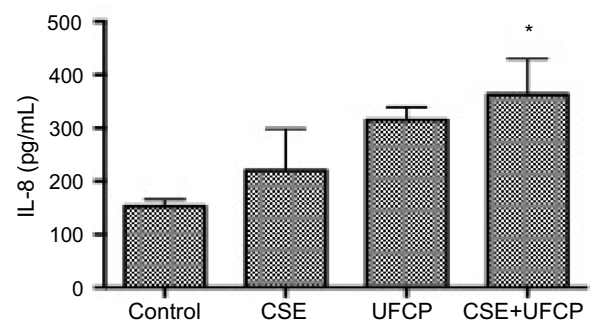

B IL-6

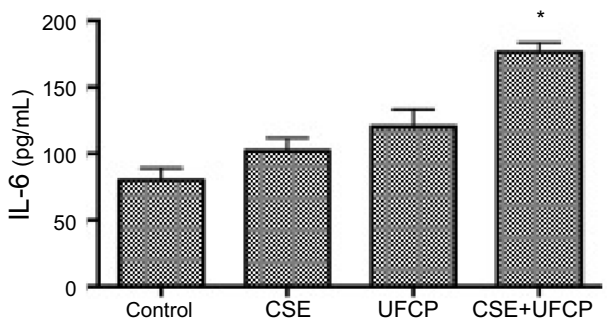

D TGF-ß1

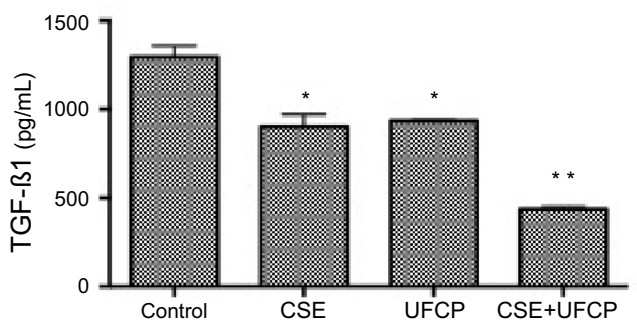

Figure 7 Effect of CSE and UFCP on production of inflammatory cytokines and growth factors. BEAS2B cells were treated with 10\% cigarette smoke extract (CSE) and/or ultrafine carbon particle (UFCP) for 24 hrs. Medium was harvested and used for quantification of cytokines and growth factors as described in the methods. (A) IL-IB; (B) IL-6; (C) IL-8; (D) TGF-ßI. Vertical axis: concentration of cytokines or growth factor $(\mathrm{pg} / \mathrm{mL})$; horizontal axis: treatment of the cells. ${ }^{*} p<0.05$; ${ }^{* *} p<0.0$ I.

deteriorating air quality and its harmful effect to the public health have been extensively attracted not only scientists, but also patients, especially patients with respiratory diseases such as COPD and asthma. In contrast to Beijing, air quality in Sanya, a city located in the South of Hainan Island, has been kept in high quality in 
China in the last decade. Consequently, senior residents and patients with COPD or asthma from Beijing temporarily migrated to Sanya city during the winter and early spring and back to Beijing during summer and early fall months.

In the current study, therefore, symptoms and lung function of the COPD patients from Beijing were compared with that of the same patients when they temporarily migrated to Sanya. We found that Sanya city air quality and relative humidity from October through March was significantly better and higher compared with that of Beijing from April through September. Consistent with air quality and relatively high humidity, COPD symptoms were significantly relieved when the patients, who lived in Beijing for their whole life, temporarily migrated to Sanya during the winter season as evidenced by improved CAT score and MMRC dyspnea scale suggesting COPD patients' exacerbation be associated with ambient air quality and relative humidity.

Consistent with our findings, previous studies have reported that $\mathrm{PM}_{2.5}$ level was significantly associated with respiratory morbidity in Beijing. ${ }^{12,13}$ It has also reported that every $10 \mu \mathrm{g} / \mathrm{m}^{3}$ increase in $\mathrm{PM}_{2.5}$ concentration was associated with an increase of $1.46 \%(95 \% \mathrm{CI}$ : $0.13-2.79 \%$ ) emergency room visit for acute exacerbation of COPD. In clinical practice, worsening of the respiratory symptoms, especially dyspnea and sputum, is the best definition of COPD exacerbations. ${ }^{14}$ In the current study, therefore, we collected records of respiratory symptoms and lung function tests in Sanya and in Beijing for the same patients and found that ambient air pollution (including particle matters) were associated with increased occurrence of respiratory symptoms in the COPD patients when they were living in Beijing, suggesting that ambient air pollution may induce adverse symptoms in COPD.

COPD patients often have emergency hospital admissions due to the exacerbation, which significantly affect patient's quality of life and even could increase COPD patients' mortality. COPD exacerbation may be associated with many risk factors. In this content, the effect of environmental exposure on COPD exacerbations has been previously reported. ${ }^{15,16}$ However, to our knowledge, longitudinal study on the association of air pollution and COPD exacerbation in same COPD patients living in different geographic locations with significantly different air quality has not been reported. Here, we report that COPD exacerbation rate and hospitalization rate were significantly reduced when the COPD patients were temporarily living in Sanya, a city with better air quality, compared with that when the patients were living in Beijing. In addition, we also noticed that dry air in Beijing might also contribute to the exacerbation of COPD in that relative humidity was year-around high in Sanya, where patients had significantly lower rate of COPD exacerbation.

The mechanisms that air pollution leads to pulmonary illnesses are so far poorly understood. Studies have shown that inflammatory mediators and reaction oxidative species (ROS) may be involved in mediating air-pollution-induced respiratory diseases. ${ }^{17,18}$ Consistently, we found that cigarette smoke extract and/or ultrafine carbon particles significantly stimulated release of pro-inflammatory cytokines, including IL-1ß, IL-6 and IL-8, by the airway epithelial cells. In contrast, TGF- $ß 1$ production was significantly inhibited by cigarette smoke and/or ultrafine carbon particles, suggesting particular matters not only induce airway inflammation, but also may suppress airway tissue repair through inhibiting synthesis of growth factors.

Previous studies have also shown that both cigarette smoke extract and ultrafine carbon black caused airway epithelial cells and lung fibroblasts DNA damage and apoptosis. ${ }^{19,20}$ Therefore, the current study further investigated potential adverse effect of cigarette smoke plus ultrafine carbon particles on human airway epithelial cell viability. Consistent with previous report, ${ }^{19,20}$ we found that either cigarette smoke or ultrafine carbon particle could induce BEAS2B cell apoptosis. We further demonstrated that cigarette smoke plus ultrafine carbon particle dramatically increased the number of apoptosis compared to that of either reagent alone, suggesting cigarette smoke plus air pollution will further cause airway cellular damage.

There were limitations in the current study. First, number of the patients enrolled into this study was limited. Second, components of the air pollutants that contribute to the exacerbation of COPD were not identified. Third, $\mathrm{PM}_{2.5}$ or $\mathrm{PM}_{10}$ isolated from air pollutant was not available and thus, ultrafine carbon particle was used to investigate $\mathrm{PM}_{2.5}$ effect. Finally, signal transduction pathways that mediate cigarette smoke and ultrafine carbon particle-induce epithelial cell apoptosis was not identified in this study. Therefore, further studies remain to be conducted.

Taken together, the current study explored the effect of air quality in two different geographic cities on the same COPD patients' symptoms, lung function and COPD exacerbation. We found that COPD symptoms were relieved, lung function was improved and COPD 
exacerbation was reduced when the COPD patients temporarily migrated from Beijing, the Capital city of China with deteriorating air quality, to Sanya city, a city with top quality ambient air in China. We proposed that ambient air quality and relative humidity are associated with the COPD exacerbation. Mechanisms of air pollutantinduced COPD exacerbation might be due to its effect on airway epithelial cell apoptosis and inflammatory injury.

\section{At a glance commentary}

\section{Scientific knowledge on the subject}

Epidemiological studies have observed association of air pollution and respiratory disease. However, association of air pollution and Chronic Obstructive Pulmonary Disease (COPD) exacerbation from the perspective of geographical air quality difference in the same COPD patients has not been reported.

\section{What this study adds to the field}

Results of this study indicated that ambient temperature, humidity and air quality are associated with quality of life in COPD patients.

\section{Acknowledgment}

This work was supported by Grant W2013BJ11 from China Healthcare Research Project. The abstract of this paper was presented at the Chest Conference "Effect of ambient air quality on exacerbation of COPD in patients and its potential mechanism" as a poster presentation.

\section{Author contributions}

All authors contributed to data analysis, drafting and revising the article, gave final approval of the version to be published, and agree to be accountable for all aspects of the work.

\section{Disclosure}

The authors report no conflicts of interest in this work.

\section{References}

1. Zhou M, He G, Fan M, et al. Smog episodes, fine particulate pollution and mortality in China. Environ Res. 2015;136:396-404. doi:10.1016/ j.envres.2014.09.038

2. Dockery DW, Pope CA, Xu X, et al. An association between air pollution and mortality in six U.S. cities. $N$ Engl J Med. 1993;329 (24):1753-1759. doi:10.1056/NEJM199312093292401

3. Dominici F, Peng RD, Bell ML, et al. Fine particulate air pollution and hospital admission for cardiovascular and respiratory diseases. Jama. 2006;295(10):1127-1134. doi:10.1001/jama.295.10.1127
4. Kunzli N, Kaiser R, Medina S, et al. Public-health impact of outdoor and traffic-related air pollution: a European assessment. Lancet. 2000;356(9232):795-801. doi:10.1016/S0140-6736(00) 02653-2

5. Lim SS, Vos T, Flaxman AD, et al. A comparative risk assessment of burden of disease and injury attributable to 67 risk factors and risk factor clusters in 21 regions, 1990-2010: a systematic analysis for the Global Burden of Disease Study 2010. Lancet. 2012;380 (9859):2224-2260. doi:10.1016/S0140-6736(12)61766-8

6. Zhao H, Li W, Gao Y, Li J, Wang H. Exposure to particular matter increases susceptibility to respiratory Staphylococcus aureus infection in rats via reducing pulmonary natural killer cells. Toxicology. 2014 Nov 5;325:180-8. doi: 10.1016/j.tox.2014.09.006

7. Dai L, Zanobetti A, Koutrakis P, Schwartz JD. Associations of fine particulate matter species with mortality in the United States: a multicity time-series analysis. Environ Health Perspect. 2014;122 (8):837-842. doi:10.1289/ehp.1307568

8. Belleudi V, Faustini A, Stafoggia M, et al. Impact of fine and ultrafine particles on emergency hospital admissions for cardiac and respiratory diseases. Epidemiology. 2010;21(3):414-423. doi:10.1097/ EDE.0b013e3181d5c021

9. Cheng MH, Chen -C-C, Chiu H-F, Yang C-Y. Fine particulate air pollution and hospital admissions for asthma: a case-crossover study in Taipei. J Toxicol Environ Health A. 2014;77(18):1075-1083. doi:10.1080/15287394.2014.922387

10. Carp H, Janoff A. Possible mechanisms of emphysema in smokers. In vitro suppression of serum elastase-inhibitory capacity by fresh cigarette smoke and its prevention by antioxidants. Am Rev Respir Dis. 1978;118(3):617-621. doi:10.1164/arrd.1978.118.3.617

11. Li L, Liu D-J. Study on an air quality evaluation model for Beijing city under haze-fog pollution based on new ambient air quality standards. Int J Environ Res Public Health. 2014;11(9):8909-8923. doi:10.3390/ijerph110908909

12. Xiong Q, Zhao W, Gong Z, Zhao W, Tang T. Fine particulate matter pollution and hospital admissions for respiratory diseases in Beijing, China. Int J Environ Res Public Health. 2015;12(9):11880-11892. doi:10.3390/ijerph120911880

13. Li P, Xin J, Wang Y, et al. The acute effects of fine particles on respiratory mortality and morbidity in Beijing, 2004-2009. Environ Sci Pollut Res Int. 2013;20(9):6433-6444. doi:10.1007/s11356-013-1688-8

14. Wedzicha JA, Singh R, Mackay AJ. Acute COPD exacerbations. Clin Chest Med. 2014;35(1):157-163. doi:10.1016/j.ccm.2013.11.001

15. Wu S, Ni Y, Li H, et al. Short-term exposure to high ambient air pollution increases airway inflammation and respiratory symptoms in chronic obstructive pulmonary disease patients in Beijing, China. Environ Int. 2016 Sep;94:76-82. doi: 10.1016/j.envint.2016.05.004

16. Xu X, Jiang SY, Wang T-Y, et al. Inflammatory response to fine particulate air pollution exposure: neutrophil versus monocyte. PLoS One. 2013;8(8):e71414. doi:10.1371/journal.pone.0071414

17. Moore E, Chatzidiakou L, Kuku M-O, et al. Global associations between air pollutants and chronic obstructive pulmonary disease hospitalizations. A systematic review. Ann Am Thorac Soc. 2016;13 (10):1814-1827. doi:10.1513/AnnalsATS.201601-064OC

18. Li J, Sun S, Tang R, et al. Major air pollutants and risk of COPD exacerbations: a systematic review and meta-analysis. Int $J$ Chron Obstruct Pulmon Dis. 2016;11:3079-3091. doi:10.2147/COPD. S122282

19. Liu X, Conner H, Kobayashi T, et al. Cigarette smoke extract induces DNA damage but not apoptosis in human bronchial epithelial cells. Am J Respir Cell Mol Biol. 2005;33(2):121-129. doi:10.1165/ rcmb.2003-03410C

20. Kim H, Liu X, Kobayashi T, et al. Reversible cigarette smoke extract-induced DNA damage in human lung fibroblasts. $\mathrm{Am}$ $J$ Respir Cell Mol Biol. 2004;31(5):483-490. doi:10.1165/ rcmb.2002-0300OC 


\section{Publish your work in this journal}

The International Journal of COPD is an international, peer-reviewed journal of therapeutics and pharmacology focusing on concise rapid reporting of clinical studies and reviews in COPD. Special focus is given to the pathophysiological processes underlying the disease, intervention programs, patient focused education, and self management protocols. This journal is indexed on PubMed Central, MedLine and CAS. The manuscript management system is completely online and includes a very quick and fair peer-review system, which is all easy to use. Visit http://www.dovepress.com/testimonials.php to read real quotes from published authors. 